\title{
Deciphering Design Principles of Förster Resonance Energy Transfer- Based Protease Substrates: Thermolysin-Like Protease from Geobacillus stearothermophilus as a Test Case
}

\author{
Sophie Ripp, ${ }^{\dagger}$ Petri Turunen, ${ }^{\dagger, \|}$ Ethan D. Minot, ${ }^{\dagger}$ Alan E. Rowan, ${ }^{\dagger, \|}$ and Kerstin G. Blank ${ }^{*}, \dagger, \S_{0}$ \\ ${ }^{\dagger}$ Institute for Molecules and Materials, Department of Molecular Materials, Radboud University, Heyendaalseweg 135,6525 AJ \\ Nijmegen, The Netherlands \\ ${ }^{\ddagger}$ Department of Physics, Oregon State University, 301 Weniger Hall, Corvallis, Oregon 97331-6507, United States \\ ${ }^{\S}$ Mechano(bio)chemistry, Max Planck Institute of Colloids and Interfaces, Potsdam-Golm Science Park, 14424 Potsdam, Germany
}

Supporting Information

\begin{abstract}
Protease activity is frequently assayed using short peptides that are equipped with a Förster resonance energy transfer (FRET) reporter system. Many frequently used donor-acceptor pairs are excited in the ultraviolet range and suffer from low extinction coefficients and quantum yields, limiting their usefulness in applications where a high sensitivity is required. A large number of alternative chromophores are available that are excited in the visible range, for example, based on xanthene or cyanine core structures. These alternatives are not only larger in size but also more hydrophobic. Here, we show that the hydrophobicity of these chromophores not only affects the solubility of the resulting FRET-

labeled peptides but also their kinetic parameters in a model enzymatic reaction. In detail, we have compared two series of 4-8 amino acid long peptides, designed to serve as substrates for the thermolysin-like protease (TLP-ste) from Geobacillus stearothermophilus. These peptides were equipped with a carboxyfluorescein donor and either Cy5 or its sulfonated derivative Alexa Fluor 647 as the acceptor. We show that the turnover rate $k_{\text {cat }}$ is largely unaffected by the choice of the acceptor fluorophore, whereas the $K_{\mathrm{M}}$ value is significantly lower for the Cy5- than for the Alexa Fluor 647-labeled substrates. TLP-ste is a rather nonspecific protease with a large number of hydrophobic amino acids surrounding the catalytic site, so that the fluorophore itself may form additional interactions with the enzyme. This hypothesis is supported by the result that the difference between Cy5- and Alexa Fluor 647-labeled substrates becomes less pronounced with increasing peptide length, that is, when the fluorophore is positioned at a larger distance from the catalytic site. These results suggest that fluorophores may become an integral part of FRET-labeled peptide substrates and that $K_{\mathrm{M}}$ and $k_{\text {cat }}$ values are generally only valid for a specific combination of the peptide sequence and FRET pair.
\end{abstract}

\section{INTRODUCTION}

Fluorescent reporter systems for the detection of protease activity consist of a spectroscopic probe with cleavable bonds. Upon cleavage, the fluorescence properties of the probe are altered, which allows for following the enzymatic reaction over time. $^{1-8}$ Three fundamentally different designs are usually utilized for the detection of protease activity: The first design consists of a fluorophore core carrying one or multiple peptide chain(s) coupled to the fluorophore via a peptide bond. , $^{3,7-12}$ Conjugation of the peptide chain(s) causes a change in the photophysical properties of the fluorophore so that the substrate is essentially nonfluorescent. The fluorescence is restored upon cleavage and is directly correlated with the enzyme activity. This design exhibits some drawbacks however. These substrates are highly artificial, as the fluorophore replaces the C-terminal part of the substrate $\left(\mathrm{P} 1^{\prime}-\mathrm{P} 4^{\prime}\right.$ subsites, according to the nomenclature of Schechter and Berger ${ }^{13}$ ). These substrates often carry two enzyme cleavable bonds, ${ }^{7,8}$ and the reaction proceeds via an intermediate with different photophysical properties, so that the reaction velocity cannot be easily determined. ${ }^{11}$ The second design makes use of selfquenching between a large number of fluorophores coupled to a model protein. 5,6 Also in this case, the substrate is hardly fluorescent, and the fluorescence signal increases once the peptide fragments are released. This substrate design is inexpensive, and one can choose from a large number of different fluorophores, including near-infrared dyes. ${ }^{6}$ These substrates contain many cleavage sites, however, leading to multistep reactions with a distribution of rate constants. ${ }^{14}$ The third design employs peptides that are equipped with a Förster resonance energy transfer (FRET) reporter system. In contrast to the first design, the peptide sequence is chosen such that it

Received: December 29, 2017

Accepted: March 19, 2018

Published: April 12, 2018 


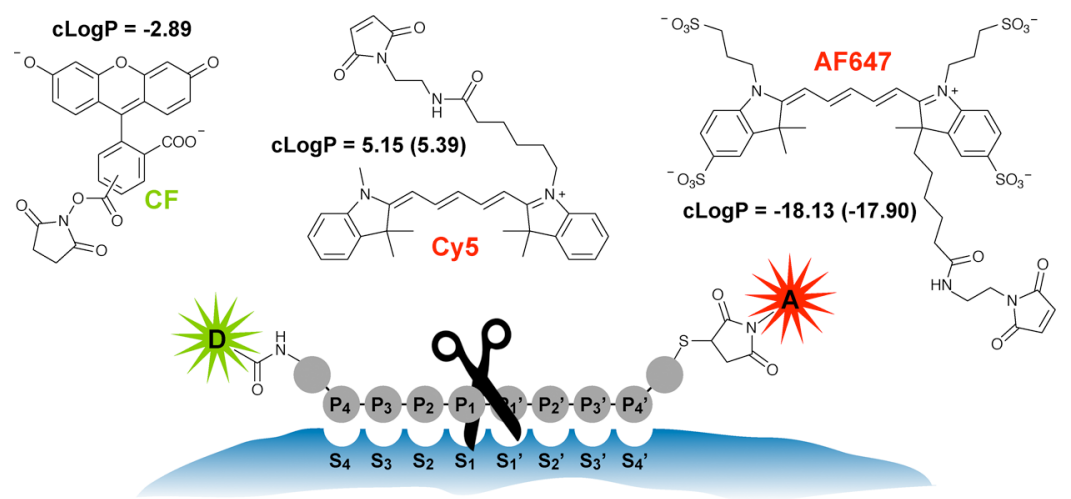

Figure 1. General structure of the FRET-labeled peptide substrates, including the FRET pairs used. An 8-amino acid-long peptide (residues P4 to $\mathrm{P}^{\prime}$ ) binds into the S4 to S4' subsites of the enzyme active site. The donor fluorophore 5,6-carboxyfluorescein [CF; N-hydroxysuccinimide (NHS)ester] was coupled to the primary amine at the N-terminus. The acceptor fluorophores, Alexa Fluor 647 (AF647) or Cy5, were coupled to a Cterminal cysteine utilizing maleimide-functionalized fluorophore derivatives. The image shows the reactive form of the fluorophores at the top. The bonds formed upon coupling are depicted together with the peptide at the bottom. The image further shows the calculated octanol-water partition coefficients $(\log P)$ for each fluorophore without and with the linker (values in brackets). CF does not contain any linker.

spans the complete recognition site from the $\mathrm{S} 4$ to $\mathrm{S} 4^{\prime}$ subsites. In the intact peptide substrate, the donor chromophore is coupled at one terminus and the acceptor chromophore at the other, allowing for efficient energy transfer. ${ }^{1,2,4,15-20}$ The fluorophores become separated upon cleavage, leading to an increase in the donor intensity. Because of the location of the cleavage site, FRET peptides are more "natural" substrates and can be utilized for all proteases, independent of the subsite specificity. More importantly, their 1:1 stoichiometry allows for accessing accurate kinetic parameters.

FRET-based substrates can be further divided into two categories: the acceptor can be "dark", serving only as a quencher, $^{4,20}$ or emit fluorescence itself. ${ }^{1,2,15-19}$ Many donoracceptor combinations have been reported. The most commonly used FRET pairs are DABCYL/EDANS ${ }^{4}$ and Abz/3-nitrotyrosine. ${ }^{21}$ Substrates using these dye combinations are easy to synthesize as the dyes can be incorporated during the solid-phase synthesis of the peptide; however, their photophysical properties (ultraviolet excitation and low quantum yield) limit their assay sensitivity. Alternatively, pairs of fluorescent proteins, such as CFP/YFP, ${ }^{15-17}$ can be used as FRET pairs. The problem with this strategy is that fluorescent proteins are bulky, potentially leading to steric hindrance upon substrate binding to the enzyme active site. Organic fluorophores that are excited in the visible range are a versatile alternative. $^{18,19}$ The large number of possible fluorophore combinations ensures that a well-suited FRET pair can be identified for the desired application.

Independent of the choice of fluorophores, designing a FRET-based substrate requires careful consideration of additional parameters such as the amino acid sequence and the peptide length. The amino acid sequence directly determines the substrate specificity as well as the hydrophobicity of the peptide. The peptide length might influence the FRET efficiency and the kinetic parameters of the enzymatic reaction. Here, we describe a systematic investigation of the influence of the peptide length on the fluorescent properties of the substrate and on the kinetics of the enzymatic reaction. As a model system to evaluate the performance of the newly designed protease substrates, we have chosen the thermolysin-like metalloprotease (TLP-ste) from Geobacillus stearothermophilus, which is a protease with a broad specificity profile. To obtain accurate kinetic parameters, the data are corrected for the inner filter effect (IFE) and compared to data where no correction has been performed. The use of two different FRET pairs (carboxyfluorescein/Cy5 and carboxyfluorescein/Alexa Fluor 647) allows for establishing general trends and for identifying specific contributions of the fluorophore molecules, for example, on substrate solubility and catalytic efficiency.

\section{RESULTS}

Substrate Design. To accurately measure the activity of TLP-ste, the first step was to design a peptide substrate functionalized with a donor-acceptor pair that is efficiently hydrolyzed by the enzyme and possesses appropriate photophysical properties. For choosing the core peptide sequence, the specificity matrix of the MEROPS peptidase database ${ }^{22}$ was utilized. We hypothesized that the specificity matrix for the homologous enzyme thermolysin ${ }^{23-25}$ would also apply for TLP-ste, as thermolysin and TLP-ste have both shown catalytic activity for similar peptide substrates. ${ }^{26,27}$ The matrix shows the most preferred amino acids for each subsite (P4 to $\mathrm{P} 4$ ', fitting into the $\mathrm{S} 4$ to $\mathrm{S}^{\prime}$ ' subsites of the enzyme active site, according to the nomenclature of Schechter and Berger, ${ }^{13}$ see Figure 1).

Using the best-scoring amino acids, the ideal sequence becomes AVAGLAGG (from P4 to P4'), with the scissile bond positioned between glycine and leucine (underlined). To increase the hydrophilicity of the peptide, the P4 alanine was replaced by serine. One additional amino acid was added at each terminus for coupling the donor and acceptor fluorophores, namely a proline at the N-terminus and a cysteine at the $\mathrm{C}$-terminus. The donor was coupled to the $\mathrm{N}$ terminal amino group of the proline using an NHS-activated fluorophore. The acceptor was coupled to the thiol group of the cysteine via thiol-maleimide conjugation (see Figure 1 and Table 1). In addition to the peptide spanning the whole recognition site (8aa), two shorter peptides (6aa and 4aa) were also synthesized (Table 1 ).

The fluorophores, forming the FRET pair, were chosen based on the following criteria: (i) both the donor and acceptor possess a high brightness and a reasonably good photostability; (ii) the photophysical properties ensure sufficient spectral overlap and consequently a sufficient FRET efficiency; (iii) spectral crosstalk is minimal; and (iv) the two dyes have a different core structure to prevent aggregation and stacking. Considering these criteria, the xanthene dye CF (maxima at 
Table 1. Substrates for TLP-ste ${ }^{a}$

\begin{tabular}{llcc}
\multicolumn{1}{c}{ name } & \multicolumn{1}{c}{ sequence $\left(\mathrm{P}_{4} \rightarrow \mathrm{P}_{4}{ }^{\prime}\right)$} & $\begin{array}{c}\text { length } \\
(\AA)^{b}\end{array}$ & $\begin{array}{c}\text { hydrophobicity } \\
(\%)^{c}\end{array}$ \\
4aa-Cy5 & CF-PAGLAC $(\mathrm{Cy5})-\mathrm{NH}_{2}$ & 22 & 67 \\
6aa-Cy5 & CF-PVAGLAGC(Cy5)-NH & 29 & 62 \\
8aa-Cy5 & CF-PSVAGLAGGC(Cy5)- & 37 & 50 \\
& $\mathrm{NH}_{2}$ & & 67 \\
4aa-AF647 & CF-PAGLAC(AF674)-NH & 22 & 50 \\
8aa-AF647 & CF-PSVAGLAGGC(AF647)- & 37 & 50 \\
& $\mathrm{NH}_{2}$ & &
\end{tabular}

${ }^{a}$ The recognition sequence is shown in bold. The cleavage site is underlined. ${ }^{b}$ Length of the fully extended peptide $(3.65 \AA /$ amino acid). ${ }^{c}$ Calculated with the peptide property calculator (www. genscript.com).

$\lambda_{\mathrm{ex}}=493 \mathrm{~nm}$ and $\lambda_{\mathrm{em}}=517 \mathrm{~nm}$ ) was chosen as the donor and a cyanine-based dye as the acceptor (Figure 1). Because of its good spectral overlap, $\mathrm{Cy} 3$ would be a suitable acceptor. On the other hand, significant spectral crosstalk with the CF donor is expected. Spectral crosstalk can lead to errors when determining the FRET efficiency and causes significant background fluorescence in the enzymatic assay. Alternatively, despite their reduced spectral overlap, Cy5 (maxima at $\lambda_{\mathrm{ex}}=$ $648 \mathrm{~nm}$ and $\lambda_{\mathrm{em}}=662 \mathrm{~nm}$ ) and its more water-soluble derivative Alexa Fluor 647 (AF647; maxima at $\lambda_{\mathrm{ex}}=650 \mathrm{~nm}$ and $\lambda_{\mathrm{em}}=664 \mathrm{~nm}$ ) were used as the acceptor fluorophore (Figure S1). ${ }^{28}$ Compared to Cy5, AF647 is sulfonated at four positions and the linker attachment site is different (Figure 1). In total, a small library of five different substrates were synthesized, using different combinations of peptide length and fluorophore pairs (Table 1).

Substrate Characterization. Prior to determining the FRET efficiency, the solubility of the substrates was tested. As shown in Table 1 , a relatively high hydrophobicity was calculated for the shorter peptides (4aa and 6aa). Coupling the hydrophobic fluorophore $\mathrm{Cy} 5$ to these peptides increases their hydrophobicity even further. To obtain information about the maximum solubility of the Cy5-labeled substrates in comparison to the AF647-labeled substrates, a dilution series $(1-10 \mu \mathrm{M})$ was prepared, and the fluorescence intensity was measured at the donor wavelength, which was also used for monitoring product formation. Provided that the substrate peptides are fully soluble, a linear relationship is expected between the fluorescence intensity and the peptide concentration. Whereas the AF647-labeled peptides show a linear relationship up to a concentration of $8 \mu \mathrm{M}$, deviations were already observed at concentrations of $5 \mu \mathrm{M}$ for the Cy5-labeled substrates (Figure S2). It has been shown earlier that Cy5 possesses a strong aggregation tendency, for example, forming $\mathrm{H}$-aggregates that are characterized by different spectroscopic properties. $^{20,28}$ In the case of our Cy5-labeled peptides, the typical spectral features of $\mathrm{H}$-aggregate formation are absent however, suggesting that aggregation is mostly driven by the peptide sequence and the overall hydrophobicity of the fluorophore-labeled peptides. Clearly, the combination of the peptide sequence with the hydrophobicity of Cy5 has a detrimental effect on peptide solubility, limiting the usable concentrations to the low micromolar range. In the following experiments, no concentrations above $5 \mu \mathrm{M}$ were used for the Cy5-labeled substrates.

The FRET efficiency $E$, which quantifies the energy transfer from the donor to the acceptor, is expressed as follows

$$
E=\frac{R_{0}{ }^{6}}{\left(R_{0}{ }^{6}+r^{6}\right)}
$$

where $R_{0}$ is the Förster radius of the FRET pair and $r$ is the DA (donor-acceptor) distance. Energy transfer is the highest when $r \ll R_{0}$ and $E$ approaches 1 . For protease assays, high FRET efficiencies close to 1 are desirable, as this allows for a straightforward quantification of the enzymatic reaction simply based on the increase in the donor intensity. Two different measurements were performed to determine $E$ for the different FRET peptides based on the donor fluorescence intensity $I$ and the donor fluorescence lifetime $\tau$. Then, $E$ can also be expressed as

$$
E=1-\frac{I_{\mathrm{D}-\mathrm{A}}}{I_{\mathrm{D}}}=1-\frac{\tau_{\mathrm{D}-\mathrm{A}}}{\tau_{\mathrm{D}}}
$$

For both measurements, the respective quantity was determined for the intact peptide $(\mathrm{D}-\mathrm{A})$ as well as for the fully cleaved peptide (D) and used to calculate $E$ (Table 2). All

Table 2. FRET Efficiencies Determined with Two Different Methods (Intensity $E_{I}$, Lifetime $\left.E_{\tau}\right)^{a}$

\begin{tabular}{lccc}
\multicolumn{1}{c}{ name } & $R_{0}(\AA)^{b}$ & $E_{I}$ & $E_{\tau}$ \\
4aa-Cy5 & 50 & $0.94 \pm 0.01$ & $0.65 \pm 0.11$ \\
6aa-Cy5 & 50 & $0.97 \pm 0.01$ & $0.77 \pm 0.18$ \\
8aa-Cy5 & 50 & $0.96 \pm 0.01$ & $0.78 \pm 0.09$ \\
4aa-AF647 & 46 & $0.98 \pm 0.01$ & $0.89 \pm 0.02$ \\
8aa-AF647 & 46 & $0.96 \pm 0.00$ & $0.87 \pm 0.01$
\end{tabular}

${ }^{a}$ Each measurement was performed in triplicate. The values represent the mean \pm the standard deviation. ${ }^{b}$ See the Supporting Information for the calculation of $R_{0}$.

$E_{I}$ values are close to 1 and vary only in the second digit. This is expected as the length of the fully extended peptide is well below the Förster radius (see Table 1 and the Supporting Information). Even though the differences in $E_{I}$ are small, a correlation with the peptide length is still observed. $E_{I}$ is slightly higher for the shorter peptides (except 4aa-Cy5, which is the least soluble peptide). This is also confirmed in the $E_{\tau}$ measurements, even though the FRET efficiencies are different between both types of measurements.

In general, the lifetime-based method is expected to be more accurate for determining the FRET efficiencies. Using the fluorescence lifetimes, the source of the emitted photons can be identified. Consequently, only photons that originate from the donor and acceptor labeled molecules are considered for the calculation of the FRET efficiency. In the presented data, however, the FRET efficiencies could not be accurately determined from the lifetime-based measurement. For FRET efficiencies $E>90 \%$, the lifetime of the donor is extremely short so that donor photons are emitted shortly after the excitation pulse. Their arrival time partially lies within the response time of the detector and might overlap with the scattered photons. In this context, an accurate fitting of the photon arrival time histogram was not possible (see the Supporting Information for a more detailed description of the lifetime experiment). In this context, no meaningful information about the FRET efficiency could be extracted from the $E_{\tau}$ measurements, even though the general trends are consistent with the intensity-based measurement. Overall, we conclude that all FRET-based substrates are functional and that the FRET efficiency is $E>95 \%$ (except 4aa-Cy5). This is a good 


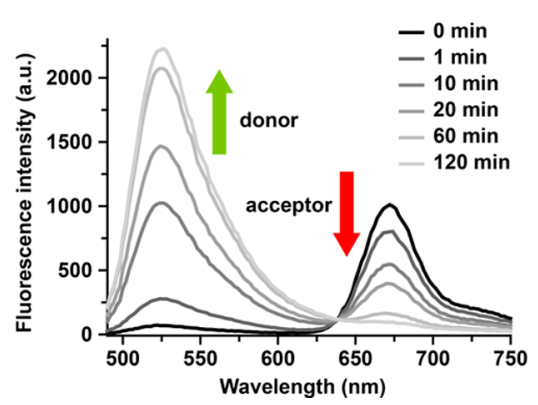

b

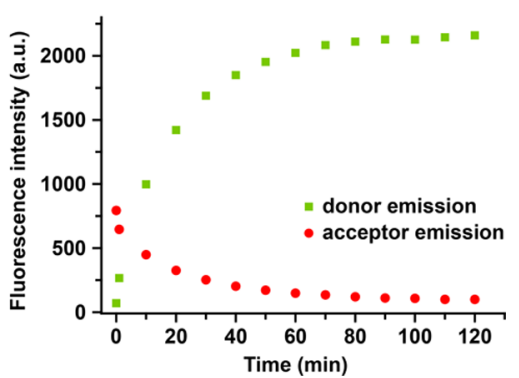

Figure 2. Enzymatic hydrolysis of the substrate 4aa-AF647 (1 $\mu \mathrm{M})$ using $30 \mathrm{nM}$ TLP-ste. (a) Emission spectra at different time points of the enzymatic reaction, recorded when the sample was excited at the donor wavelength $\left(\lambda_{\mathrm{ex}}=450 \mathrm{~nm}\right)$. (b) Progress curve of the enzymatic reaction, following both donor $\left(\lambda_{\mathrm{ex}}=450 \mathrm{~nm}\right.$ and $\left.\lambda_{\mathrm{em}}=520 \mathrm{~nm}\right)$ and acceptor emission $\left(\lambda_{\mathrm{ex}}=450 \mathrm{~nm}\right.$ and $\left.\lambda_{\mathrm{em}}=660 \mathrm{~nm}\right)$.

starting point for the kinetic measurements where the increase in the donor signal is monitored.

Kinetic Measurements. To test which substrate design yields the best probe for measuring the protease activity, the five substrates were used in kinetic measurements using TLPste. In a first series of experiments, TLP-ste was added to the different substrate solutions, and both donor and acceptor intensities were followed as a function of time. Figure 2 shows the result obtained for the substrate 4aa-AF647 as an example. At $t=0 \mathrm{~min}$, most of the emitted photons originated from the acceptor $\left(\lambda_{\mathrm{em}}=660 \mathrm{~nm}\right)$. As the reaction proceeded, the intensity of the acceptor decreased while the donor intensity increased. When following the enzymatic progress curve at the donor and acceptor wavelengths, a typical saturation curve was obtained. This clearly indicates that the substrate is enzymatically cleaved, validating the choice of the amino acid sequence. Additional proof for the specificity of the reaction was obtained from two experiments where the substrate was either incubated in pure buffer or with an inactive enzyme [preincubated with the inhibitor ethylenediaminetetraacetic acid (EDTA)]. In both cases, no or only a very small increase in donor fluorescence was observed (Figures S4 and S5).

The next step was to determine the kinetic constants, $K_{\mathrm{M}}$ and $v_{\max }$ as described by the Michaelis-Menten equation

$$
v=\frac{v_{\max }[\mathrm{S}]}{K_{\mathrm{M}}+[\mathrm{S}]}
$$

where $v$ is the reaction velocity, $v_{\max }$ is the maximum velocity, [S] is the substrate concentration, and $K_{\mathrm{M}}$ is the MichaelisMenten constant. The reaction velocity, which was obtained from the initial slope of the donor progress curve, describes the increase in the product concentration over time. To obtain the product concentration, a calibration curve is required that relates the measured fluorescence intensity of the donor to the concentration of the respective donor-carrying product. When using FRET-based substrates, two complications arise when determining the calibration factor. As the FRET efficiency is not $100 \%$, the donor signal will not increase from 0 to $100 \%$ upon cleavage, as it is usually assumed when preparing the calibration curve. Instead, the donor intensity will only increase from 5 to $100 \%$ (assuming a FRET efficiency of 95\%). It is consequently required to correct the calibration factor by the FRET efficiency. In addition to the FRET efficiency, also the so-called IFE needs to be considered for an accurate calibration. $^{29-31}$ The high substrate concentrations, required for the kinetic measurements, result in a large number of absorbing chromophores in solution. This might cause inhomogeneities in the excitation intensity throughout the sample so that not all fluorophores are excited. Further, some of the emitted light might be reabsorbed by the surrounding chromophores (donor and/or acceptor) so that it cannot reach the detector. As a consequence, the apparent fluorescence intensity is diminished, leading to a nonlinear relationship between the product concentration and the fluorescence signal and, ultimately, to an underestimation of the real enzymatic rate. To correct for the IFE in the kinetic data, the measured fluorescence intensities $F_{\text {meas }}$ need to be multiplied with a correction factor $\mathrm{CF}_{\mathrm{IFE}}$ (see the Supporting Information for the experimentally derived correction factors; text and Figure S6). The results of the kinetic analysis after employing corrections for the FRET efficiency and the IFE are summarized in Table 3.

Table 3. Kinetic Parameters of the Five Different Substrates, Corrected for IFE and FRET Efficiency ${ }^{a}$

\begin{tabular}{lcccc}
\multicolumn{1}{c}{ name } & $K_{\mathrm{M}}(\mu \mathrm{M})$ & $\begin{array}{c}v_{\max } \\
\left(\times 10^{-4} \mu \mathrm{M} \mathrm{s}^{-1}\right)\end{array}$ & $\begin{array}{c}k_{\text {cat }} \\
\left(\mathrm{s}^{-1}\right)\end{array}$ & $\begin{array}{c}k_{k_{\text {cat }} / K_{\mathrm{M}}} \\
\left(\mathrm{s}^{-1} \mathrm{M}^{-1}\right)\end{array}$ \\
\hline 4aa-Cy5 & $2.4 \pm 1.9$ & $3.7 \pm 1.6$ & 0.37 & 154200 \\
6aa-Cy5 & $11.6 \pm 13.0$ & $29.6 \pm 26.6$ & 2.96 & 255200 \\
8aa-Cy5 & $17.6 \pm 11.1$ & $78.2 \pm 48.2$ & 7.82 & 444300 \\
4aa-AF647 & $53.3 \pm 33.9$ & $7.0 \pm 3.8$ & 0.70 & 13100 \\
8aa-AF647 & $77.6 \pm 38.5$ & $57.0 \pm 27.0$ & 5.70 & 73500
\end{tabular}

${ }^{a}$ Each measurement was performed in triplicate. The values were obtained from fitting the data to the Michaelis-Menten equation and represent the mean \pm the standard deviation.

For comparison, the kinetic constants obtained without applying the correction(s) are shown in Table S3. Representative Michaelis-Menten and Eadie-Hofstee plots utilizing both corrections are also shown in the Supporting Information (Figure S7).

As expected for substrates with a FRET efficiency close to 1, the FRET efficiency correction has only a minor effect on $K_{\mathrm{M}}$ and $v_{\max }$. A much larger effect is observed for the IFE correction (Table S3). Palmier et al. ${ }^{31}$ reported that the IFE cannot be ignored when the sum of the absorbance values at the donor excitation $\left(A_{490 \mathrm{~nm}}\right)$ and emission wavelengths $\left(A_{520 \mathrm{~nm}}\right)$ exceeds 0.08 , that is, $\mathrm{CF}_{\mathrm{IFE}}>1.1$, disregarding the path length. In our experiments, we observe the largest effect of the IFE correction for the substrates with the highest $K_{\mathrm{M}}$ (4aa-AF647 and 8aaAF647). For these substrates, the IFE affects the measured reaction velocities already at substrate concentrations far below $K_{\mathrm{M}}$ so that the correction has a larger effect on the overall shape of the Michaelis-Menten curve and the resulting kinetic parameters. Interestingly, the IFE correction has only a minor 


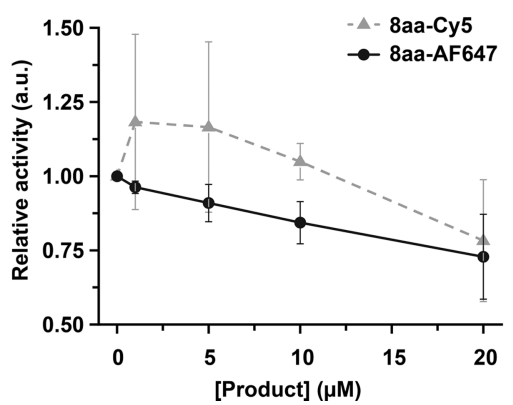

b

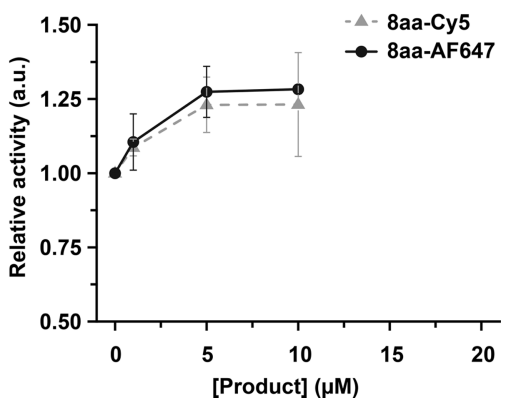

Figure 3. Activity measurements performed in the presence of different concentrations of the product. (a) Measurement performed in the presence of the acceptor-labeled C-terminal product H-LAGGC(AF647)- $\mathrm{NH}_{2}$. (b) Measurement performed in the presence of the nonfluorescent $\mathrm{N}$ - and Cterminal products, acetyl-PSVAG-OH and H-LAGGC- $\mathrm{NH}_{2}$. All measurements were performed in triplicate. The values represent the mean \pm the standard deviation.

effect on the $k_{\text {cat }} / K_{\mathrm{M}}$ values, which describe the initial phase of the reaction where the product concentration is still low. In the following, we will only discuss the values obtained when applying both the correction factors.

For all substrates, the obtained $K_{\mathrm{M}}$ values lie above the highest concentrations used for the kinetic measurements (with the exception of 4 aa-Cy5). As a consequence, the $K_{\mathrm{M}}$ and $k_{\text {cat }}$ values are considered to have a relatively large error. We are still reporting $K_{\mathrm{M}}$ and $k_{\text {cat }}$, as several important trends can be observed for the different substrates. We have also determined $K_{\mathrm{M}}$ and $k_{\text {cat }}$ independently from Eadie-Hofstee plots (Figure S7 and Table S4) and observe a reasonable agreement between the two different fitting methods. In addition, the $k_{\text {cat }} / K_{\mathrm{M}}$ values were calculated from $K_{\mathrm{M}}$ and $k_{\mathrm{cat}}$ and obtained directly from the initial slope of the Michaelis-Menten graph. Both values show good agreement (Table S5).

When comparing $k_{\text {cat }}$ for the different substrates, little difference is observed for the corresponding Cy5- and AF647labeled substrates (less than factor 2), suggesting that the choice of the fluorophore does not influence the rate of the catalytic reaction itself. By contrast, the peptide length has a strong effect on $k_{\text {cat }}$ : the longer the substrate, the higher the $k_{\text {cat }}$ (21-fold from 4aa-Cy5 to 8aa-Cy5 and 8-fold from 4aa-AF647 to 8aa-AF647). The $K_{M}$ values are affected by both the peptide length and the choice of the fluorophore. Much lower $K_{M}$ values are observed for the Cy5-labeled peptides (22-fold for $4 \mathrm{aa}$ and 4-fold for 8aa), suggesting that the Cy5-based substrates bind stronger into the enzyme active site. $K_{\mathrm{M}}$ increases with the peptide length, showing a 7 -fold increase for the Cy5 substrates and a 1.5-fold increase for the AF647 substrates. The higher $K_{M}$ values observed for longer peptides are surprising. One would intuitively expect that a longer recognition sequence would enhance the affinity and thus lower the $K_{\mathrm{M}}$. Overall, the effect on $K_{\mathrm{M}}$ is stronger for the Cy5-labeled peptides, suggesting that the fluorophores themselves contribute to the interaction between the substrate and the enzyme active site. Alternatively, rebinding of product molecules into the active site is another possible explanation for the higher $K_{M}$ of the longer substrates. If product molecules were able to rebind to the active site, they would have to be considered as competitive inhibitors so that only apparent $K_{\mathrm{M}}$ values can be determined.

Product Inhibition. The Gly-Leu bond underlined in Table 1 is the most probable cleavage site. As TLP-ste is a relatively nonspecific protease, however, it cannot be excluded that the enzyme is able to cleave the substrate peptides at different positions. If this were the case, rebinding (eventually followed by proteolytic cleavage) would be a lot more likely for the longer substrates (6aa-Cy5, 8aa-Cy5, and 8aa-AF647), and it would explain the higher $K_{\mathrm{M}}$ observed for these substrates. Using the 8aa-Cy5 and 8aa-AF647 substrates and their corresponding products, we investigated the extent of rebinding (product inhibition) by measuring the enzyme activity in the presence of increasing product concentrations (Figure 3). As the addition of the donor-carrying product would cause a very high initial fluorescence intensity and would impair the detection of product formation in the first minutes of the kinetic measurement, only the acceptor-carrying product $\mathrm{H}$ LAGGC(AF647)- $\mathrm{NH}_{2}$ was used. For practical reasons, the AF647-carrying product was used for assaying both the AF647 and $\mathrm{Cy} 5$ substrates. The absence of the donor-carrying product in the assay misrepresents the real situation of a kinetic measurement, however. Consequently, we also measured the product inhibition using a mixture of the unlabeled products (acetyl-PSVAG-OH and H-LAGGC-NH $\mathrm{N}_{2}$ ). The extent of inhibition was estimated using the relative fraction of enzyme activity (activity in the absence of the product divided by the activity in the presence of the product).

Figure $3 a$ shows that the relative activity decreases as the concentration of acceptor-carrying product increases, suggesting that product inhibition takes place. This effect is not observed, however, when performing the measurement with the nonfluorescent products (Figure 3b). The decrease in the relative activity observed for the AF647-carrying product (Figure 3a) might be due to the IFE or intermolecular FRET, as a significant number of chromophores has been added to the solution. Considering the data from both measurements and their corresponding error, it can be concluded that product inhibition does not have a strong effect on the catalytic reaction. Therefore, the higher $K_{\mathrm{M}}$ observed for longer substrates cannot be explained by product inhibition. This indirectly supports our alternative hypothesis that the acceptor fluorophore contributes to substrate binding into the active site, thereby affecting the $K_{\mathrm{M}}$ value of the enzymatic reaction.

\section{DISCUSSION}

The aim of this study was to investigate the importance of the peptide length and fluorophore choice as design parameters for the development of FRET-based substrates for protease assays. The peptide length is expected to affect many substrate 
characteristics simultaneously, such as the FRET efficiency, substrate solubility, binding affinity, and catalytic rate as well as possible product inhibition and enzyme-fluorophore interactions. Indeed, our results show that all of these characteristics are affected, some in opposing ways. For example, when increasing the peptide length, the FRET efficiency decreases slightly, whereas the solubility increases. These effects are small, however, when compared to the influence of the peptide length on the kinetic constants and on the enzyme-fluorophore interactions.

For both the CF/Cy5 and CF/AF647 series, an increase in $k_{\text {cat }}$ was observed when extending the length of the peptide. A similar length dependence was also observed for the serine protease chymotrypsin and was explained with a dynamic coupling between subsite interactions and catalysis. ${ }^{32}$ Even though the catalytic mechanisms of TLP-ste and chymotrypsin are different, the substrate binding site(s) seems to cooperatively communicate with the active site in both cases. When comparing the $k_{\text {cat }}$ values measured here $\left(0.37-7.8 \mathrm{~s}^{-1}\right)$ with other results obtained for TLP-ste or thermolysin, previously reported values appear to be higher $\left(2-5200 \mathrm{~s}^{-1}\right) .^{27,33-36}$ Most substrates characterized earlier span maximally the $\mathrm{S} 2-\mathrm{S} 2$ ' subsites. $^{33,34,36}$ Considering the observed length dependence, even higher $k_{\text {cat }}$ values may therefore be expected for the longer substrates reported here. A direct extrapolation is difficult, however. The previously reported substrates possess different peptide sequences and/ or fluorescent reporter systems with unknown cooperativity effects. $^{33,34,36}$

Although increasing peptide length has a positive effect on $k_{\text {cat }}$, the opposite is the case for $K_{\mathrm{M}}$. Assuming that $K_{\mathrm{M}}$ reflects the affinity of the enzyme for the substrate, the enzymesubstrate interaction seems to become weaker with increasing peptide length. This result is not only unexpected but also in contrast with the results obtained for chymotrypsin, where $K_{M}$ tends to decrease with increasing peptide length. ${ }^{32}$ As our control experiments do not show any evidence for product inhibition, we conclude that the fluorophore itself contributes to this effect. As a direct result of its sulfonation, AF647 is less hydrophobic and bulkier than Cy5 (Figure 1). When inspecting the geometry of the thermolysin substrate binding site, a $4 \mathrm{~nm}$ long cleft is observed (Figure S8). This cleft can accommodate at least five $\mathrm{N}$-terminal and $\mathrm{C}$-terminal amino acids from the cleavage site. For the shorter substrates, it is therefore likely that the fluorophores interact with this substrate binding cleft. The $S 1^{\prime}-S 4^{\prime}$ subsites contain a large number of hydrophobic amino acids, which may contribute to the increased affinity for Cy5 over AF647. Even though the AF647-labeled substrates possess a higher $K_{\mathrm{M}}$ than the Cy5-labeled substrates, the measured values are still lower than most $K_{\mathrm{M}}$ values reported in the literature. ${ }^{27,33-35}$ The only other substrate that shows a $K_{\mathrm{M}}$ value lower than $100 \mu \mathrm{M}$ is the 4 -amino-acid long sequence GlyPheLeuAla, which carries the fluorophores dabsyl and bimane as a FRET pair. ${ }^{36}$ Interestingly, this sequence also possesses a $k_{\text {cat }}$ similar to the values measured here.

Our results suggest that both the peptide length and the nature of the acceptor fluorophore determine the kinetic constants of the enzymatic reaction. The peptide length has a direct effect on $k_{\text {cat }}$ and $K_{\mathrm{M}}$. The peptide length further influences $K_{\mathrm{M}}$ indirectly, positioning the acceptor fluorophore relative to the binding cleft and thereby modulating its interaction with the enzyme. $k_{\text {cat }}$ and $K_{\mathrm{M}}$ affect the catalytic efficiency $k_{\text {cat }} / K_{\mathrm{M}}$ in opposite directions; however, the increase in $k_{\text {cat }}$ overcompensates the negative effect on $K_{\mathrm{M}}$ so that an overall increase in the catalytic efficiency is observed with increasing peptide length. The $k_{\text {cat }} / K_{\mathrm{M}}$ values lie in the range from $10^{4}$ to $10^{6} \mathrm{M}^{-1} \mathrm{~s}^{-1}$, which are in the range of previously reported values for enzymes from the thermolysin family. ${ }^{27,33-36}$ The clear influence of the fluorophore on $K_{\mathrm{M}}$ argues that the catalytic efficiency could be increased further when using more hydrophobic amino acids in the corresponding subsites. As this would have a negative effect on substrate solubility, however, this would have to be compensated for in other subsites. Considering that little information is available on possible cooperative effects between subsites, this goal may be difficult to achieve with a rational approach. A combinatorial library, using the consensus sequence as a starting point, may be a promising strategy toward better performing sequences.

It appears likely that the fluorophore itself causes the difference in the $K_{\mathrm{M}}$ values; however, the linker between the fluorophore and the reactive maleimide functionality cannot be ignored. The linkers attached to $\mathrm{Cy} 5$ and AF647 are identical, but they are coupled to the fluorophore at different positions (Figure 1). They may therefore orient the fluorophores differently with respect to the hydrophobic binding site. Also, the linkers themselves contain a hydrophobic hydrocarbon chain, which may further influence the interaction between the fluorophore and the enzyme. Most likely, the combination of the linker and the fluorophore contributes to favorable or unfavorable enzyme-substrate interactions, thereby increasing or decreasing the $K_{\mathrm{M}}$ value. We argue that such contributions from fluorophores and/or linkers will be generally observed also for other fluorophores and proteases and are not necessarily restricted to hydrophobic interactions. Different kinetic constants have, for example, also been determined for $\alpha$ chymotrypsin when measured with succinyl-AAPF- $p$-nitroanilide ${ }^{32}$ and succinyl-AAPF-morpholinecarbonyl-rhodamine $110 .{ }^{11}$ In addition, many fluorophores have been shown to interact nonspecifically with $\mathrm{DNA},{ }^{37}$ polypeptides, ${ }^{38}$ and lipid membranes, ${ }^{39}$ suggesting that such interactions frequently occur for many different biological systems. To obtain a better, structure-based understanding of (nonspecific) enzymefluorophore interactions, modeling combined with molecular dynamics simulations may be the method of choice. Simulations of FRET-labeled proteins are now frequently employed to establish the conformational space of the attached fluorophores and to accurately determine molecular distances. $^{38,40}$ These methods may also be suited to obtain detailed structural insights into possible linker orientations and enzyme-fluorophore interactions.

Overall, our results suggest that long peptides spanning all S4-S4' subsites should be preferred, even for rather nonspecific proteases, such as those from the thermolysin family. This not only increases the catalytic efficiency but also provides more possibilities for tuning the hydrophilicity of the peptide sequence. Most importantly, it will allow for reducing possible enzyme-fluorophore interactions, as the distance between the fluorophore and the catalytic site increases. In cases where this is not possible, for example, when the product rebinds to the enzyme as an inhibitor or substrate, the influence of fluorophores and linkers needs to be considered and should ideally be tested in a control experiment. In this control experiment, a different fluorophore can be used, as shown here, or the position of the donor and acceptor can simply be exchanged on the same peptide sequence. 


\section{CONCLUSIONS}

In conclusion, we have shown that peptides labeled with a carboxyfluorescein donor and a Cy5 or Alexa Fluor 647 acceptor can serve as powerful enzyme substrates for in vitro kinetic measurements of the protease TLP-ste. Coupling these FRET pairs to the consensus recognition sequence yields useful enzyme substrates that allow for determining the kinetic constants of the enzymatic reaction. Our results suggest that the fluorophore and its linker become integral parts of the substrate molecule, contributing to the enzyme-substrate interaction. The possibility of enzyme-fluorophore interactions needs to be considered when kinetic constants are to be obtained. Protease substrates that use bright organic fluorophores as FRET pairs may find use in applications where a high sensitivity is required, for example, in miniaturized and parallelized assays for specificity profiling, inhibitor screening, enzyme optimization, diagnostics, ${ }^{19,41-44}$ and eventually for single molecule enzymology. ${ }^{45,46}$ Using a nearinfrared emitting acceptor will further allow for using the substrates in cell-based or in vivo studies, for example, when fusing the substrate to a cell-penetrating peptide. ${ }^{47,48}$

Experimental Procedures. Materials. The FRET-labeled peptides were synthesized by JPT Peptide Technologies (Berlin, Germany). Following solid-phase synthesis of the peptides, the donor fluorophore $\mathrm{CF}$ was coupled to the $\mathrm{N}$ terminal amine of the proline residue via NHS-ester chemistry. The acceptor fluorophores Cy5 or Alexa Fluor 647 were coupled to the C-terminal cysteine using maleimide-activated fluorophores. The high-performance liquid chromatographypurified and freeze-dried substrate peptides, containing acetate as the counterion, were dissolved in a concentration of $2 \mathrm{mM}$ in dimethyl sulfoxide (DMSO) and stored in aliquots at $-20{ }^{\circ} \mathrm{C}$. The enzyme TLP-ste (C288L/N181C) was expressed and purified as described. ${ }^{49}$ Assayed with $N$-(3-[2-Furyl] acryloyl)Gly-Leu amide, this enzyme preparation possesses a $K_{M}$ value of $7 \mathrm{mM}$ and a $k_{\text {cat }}$ value of $14 \mathrm{~s}^{-1}$.

Determination of the FRET Efficiency. The FRET efficiency was determined from the fluorescence intensities and lifetimes of the intact substrates (donor fluorescence in the presence of the acceptor) and the cleaved substrates (donor fluorescence in the absence of the acceptor). The substrate stock solutions were diluted to $1 \mu \mathrm{M}$ in MOPS buffer $(20 \mathrm{mM}$ MOPS pH 7.4, $5 \mathrm{mM} \mathrm{CaCl}_{2}$ ) with $10 \%$ DMSO. The cleaved substrates were prepared by adding $1 \mathrm{nM}$ TLP-ste to the substrate solutions. These samples were incubated at room temperature in the dark for at least $16 \mathrm{~h}$ to ensure complete hydrolysis. For the intensity-based measurement, the fluorescence intensity of each sample was measured $\left(\lambda_{\mathrm{ex}}=450 \mathrm{~nm}\right.$ and $\lambda_{\text {em }}=520 \mathrm{~nm}$ ) with a microplate reader (Infinite 200 Pro, Tecan) using black, flat-bottom 96-well plates (Greiner). For the lifetime-based measurement, the substrate solutions were analyzed using a confocal microscope equipped with a pulsed $485 \mathrm{~nm}$ laser and an avalanche photodiode detector to allow for time-correlated single photon counting (see the Supporting Information).

Kinetic Measurements. The kinetic measurements were performed in a microplate reader. The substrates were diluted in MOPS buffer ensuring a final concentration of $10 \%$ DMSO in all samples. The substrate concentrations covered a range between 0 and $5 \mu \mathrm{M}$ for the Cy5-based substrates and between 0 and $8 \mu \mathrm{M}$ for the AF647-based substrates. One nanomolar concentration of the enzyme TLP-ste was used. The measure- ment was started by adding $10 \mu \mathrm{L}$ of enzyme to $190 \mu \mathrm{L}$ of the substrate solution. Product formation was monitored at the donor wavelength $\left(\lambda_{\mathrm{ex}}=490 \mathrm{~nm}\right.$ and $\left.\lambda_{\mathrm{em}}=520 \mathrm{~nm}\right)$. The reaction was followed for $1 \mathrm{~h}(90 \mathrm{~s}$ intervals between two data points) at a temperature of $30^{\circ} \mathrm{C}$.

The fluorescence intensity was converted into product concentration using a calibration curve. The calibration curve was determined using the donor-carrying product (CF-PSVAG$\mathrm{NH}_{2}$ ). The product was diluted in MOPS buffer with $10 \%$ DMSO, covering a concentration range from 0 to $4 \mu \mathrm{M}$. The corresponding fluorescence intensity values were measured with the microplate reader using exactly the same settings as for the kinetic measurements. The data were fitted to a straight line in the concentration range from 0 to $2 \mu \mathrm{M}$ ( 8 data points). The conversion factor used for all kinetic measurements represents the mean of four independent measurements.

All kinetic measurements were performed in triplicate, and the data were analyzed in the following way: The slope of the initial linear part of the kinetic curve was determined using least-squares fitting in OriginLab 8.6. When desired, the slope was corrected for the FRET efficiency (division of the slope by the FRET efficiency) and/or for the IFE (multiplication of the slope by the IFE correction factor). The slope was then converted into the reaction velocity $(\mu \mathrm{M}$ of product per second) using the previously determined calibration factor. The data derived from each experiment were plotted in the Michaelis-Menten and Eadie-Hofstee form. These plots were fitted to obtain the $K_{\mathrm{M}}$ and $v_{\max }$ values. Subsequently, the mean values and standard deviations were calculated. $k_{\text {cat }}$ was calculated by dividing $v_{\max }$ by the enzyme concentration. $k_{\text {cat }} / K_{\mathrm{M}}$ was calculated from the obtained $k_{\text {cat }}$ and $K_{\mathrm{M}}$ values. It was confirmed by calculating the slope of the linear part of the Michaelis-Menten plot (when $[S] \ll K_{\mathrm{M}}$ ). A comparison of the $k_{\text {cat }} / K_{\mathrm{M}}$ values is shown in the Supporting Information (Table S5).

Product Inhibition. Peptides representing both the Nterminal (acetyl-PSVAG-OH, acetylated N-terminus, and free C-terminus) and C-terminal (H-LAGGC- $\mathrm{NH}_{2}$, free $\mathrm{N}$ terminus, and amidated $\mathrm{C}$-terminus) reaction products of the 8 aa substrates were used to test for product inhibition. The kinetic measurements were performed with substrate concentrations ranging between 0 and $2 \mu \mathrm{M}$, to which different concentrations of the product $(0-20 \mu \mathrm{M})$ were added. In one series of measurements, the nonfluorescent products (acetylPSVAG-OH and H-LAGGC- $\mathrm{NH}_{2}$ ) were added. The measurement was repeated using only the acceptor-bearing C-terminal product $\mathrm{H}$-LAGGC(AF647)- $\mathrm{NH}_{2}$. The donor-carrying $\mathrm{N}$ terminal product was not used, as it would cause a very strong background fluorescence, preventing the detection of enzyme activity. The extent of product inhibition was estimated by comparing the reaction velocity in the absence and presence of the product. Each measurement was performed in triplicate.

\section{ASSOCIATED CONTENT}

\section{Supporting Information}

The Supporting Information is available free of charge on the ACS Publications website at DOI: 10.1021/acsomega.7b02084.

Additional characterization data of the FRET peptides, IFE correction, and the active site structure of the homologue thermolysin (PDF) 


\section{AUTHOR INFORMATION}

\section{Corresponding Author}

*E-mail: kerstin.blank@mpikg.mpg.de (K.G.B.).

\section{ORCID $\odot$}

Kerstin G. Blank: 0000-0001-5410-6984

\section{Present Address}

"University of Queensland, Australian Institute for Bioengineering and Nanotechnology, Corner College and Cooper Rds, Brisbane, Queensland 4072, Australia.

\section{Notes}

The authors declare no competing financial interest.

\section{ACKNOWLEDGMENTS}

The authors thank Bertus van den Burg for providing TLP-ste expressing strains, Johanna Mansfeld for help with TLP-ste expression and purification, Martin Höfling for his initial advice when choosing the donor-acceptor pairs, and Reinhild Dünnebacke for performing the EDTA inhibition experiment. This work was supported by the Human Frontier Science Program (grant RGY0058/2010, K.G.B. and E.D.M.), the Netherlands Organization for Scientific Research (grant 700.58.430 K.G.B.), and the Dutch National Research School Combination Catalysis Controlled by Chemical Design (grant 2009-10016B, A.E.R.).

\section{REFERENCES}

(1) Latt, S. A.; Auld, D. S.; Vallee, B. L. Fluorescence determination of carboxypeptidase A activity based on electronic energy transfer. Anal. Biochem. 1972, 50, 56-62.

(2) Carmel, A.; Zur, M.; Yaron, A.; Katchalski, E. Use of substrates with fluorescent donor and acceptor chromophores for the kinetic assay of hydrolases. FEBS Lett. 1973, 30, 11-14.

(3) Zimmerman, M.; Ashe, B.; Yurewicz, E. C.; Patel, G. Sensitive assays for trypsin, elastase, and chymotrypsin using new fluorogenic substrates. Anal. Biochem. 1977, 78, 47-51.

(4) Matayoshi, E.; Wang, G.; Krafft, G.; Erickson, J. Novel fluorogenic substrates for assaying retroviral proteases by resonance energy transfer. Science 1990, 247, 954-958.

(5) Jones, L. J.; Upson, R. H.; Haugland, R. P.; Panchuk-Voloshina, N.; Zhou, M.; Haugland, R. P. Quenched BODIPY Dye-Labeled Casein Substrates for the Assay of Protease Activity by Direct Fluorescence Measurement. Anal. Biochem. 1997, 251, 144-152.

(6) Weissleder, R.; Tung, C.-H.; Mahmood, U.; Bogdanov, A. In vivo imaging of tumors with protease-activated near-infrared fluorescent probes. Nat. Biotechnol. 1999, 17, 375-378.

(7) Leytus, S. P.; Melhado, L. L.; Mangel, W. F. Rhodamine-based compounds as fluorogenic substrates for serine proteinases. Biochem. J. 1983, 209, 299-307.

(8) Boonacker, E.; Elferink, S.; Bardai, A.; Fleischer, B.; Van Noorden, C. J. F. Fluorogenic Substrate [Ala-Pro]2-Cresyl Violet But Not Ala-Pro-Rhodamine 110 Is Cleaved Specifically by DPPIV Activity: A Study in Living Jurkat Cells and CD26/DPPIV-transfected Jurkat Cells. J. Histochem. Cytochem. 2003, 51, 959-968.

(9) Wang, Z.-Q.; Liao, J.; Diwu, Z. N-DEVD-N'-morpholinecarbonyl-rhodamine 110: novel caspase-3 fluorogenic substrates for cell-based apoptosis assay. Bioorg. Med. Chem. Lett. 2005, 15, 2335-2338.

(10) Li, J.; Yao, S. Q. "Singapore Green”: A New Fluorescent Dye for Microarray and Bioimaging Applications. Org. Lett. 2009, 11, 405408.

(11) Terentyeva, T. G.; Van Rossom, W.; Van der Auweraer, M.; Blank, K.; Hofkens, J. Morpholinecarbonyl-Rhodamine 110 Based Substrates for the Determination of Protease Activity with Accurate Kinetic Parameters. Bioconjugate Chem. 2011, 22, 1932-1938.

(12) Sakabe, M.; Asanuma, D.; Kamiya, M.; Iwatate, R. J.; Hanaoka, K.; Terai, T.; Nagano, T.; Urano, Y. Rational Design of Highly
Sensitive Fluorescence Probes for Protease and Glycosidase Based on Precisely Controlled Spirocyclization. J. Am. Chem. Soc. 2013, 135, 409-414.

(13) Schechter, I.; Berger, A. On the size of the active site in proteases. I. Papain. Biochem. Biophys. Res. Commun. 1967, 27, 157162.

(14) Vorob'ev, M. M. Quantification of two-step proteolysis model with consecutive demasking and hydrolysis of peptide bonds using casein hydrolysis by chymotrypsin. Biochem. Eng. J. 2013, 74, 60-68.

(15) Mitra, R. D.; Silva, C. M.; Youvan, D. C. Fluorescence resonance energy transfer between blue-emitting and red-shifted excitation derivatives of the green fluorescent protein. Gene 1996, 173, 13-17.

(16) Xu, X.; Gerard, A. L. V.; Huang, B. C. B.; Anderson, D. C.; Payan, D. G.; Luo, Y. Detection of programmed cell death using fluorescence energy transfer. Nucleic Acids Res. 1998, 26, 2034-2035.

(17) Mahajan, N. P.; Harrison-Shostak, D. C.; Michaux, J.; Herman, B. Novel mutant green fluorescent protein protease substrates reveal the activation of specific caspases during apoptosis. Chem. Biol. 1999, $6,401-409$

(18) George, J.; Teear, M. L.; Norey, C. G.; Burns, D. D. Evaluation of an Imaging Platform during the Development of a FRET Protease Assay. J. Biomol. Screening 2003, 8, 72-80.

(19) Díaz-Mochón, J. J.; Bialy, L.; Bradley, M. Dual colour, microarray-based, analysis of 10000 protease substrates. Chem. Commun. 2006, 3984-3986.

(20) Lebel, R.; Bonin, M.-A.; Zriba, R.; Radulska, A.; Neugebauer, $\mathrm{W}$; Lepage, M. Impact of $\mathrm{H}$-aggregation on activatable MMP-2specific probes for optical imaging. Contrast Media Mol. Imaging 2012, 7, 328-337.

(21) Meldal, M.; Breddam, K. Anthranilamide and nitrotyrosine as a donor-acceptor pair in internally quenched fluorescent substrates for endopeptidases: Multicolumn peptide synthesis of enzyme substrates for subtilisin carlsberg and pepsin. Anal. Biochem. 1991, 195, 141-147.

(22) Rawlings, N. D.; Barrett, A. J.; Bateman, A. MEROPS: the database of proteolytic enzymes, their substrates and inhibitors. Nucleic Acids Res. 2012, 40, D343-D350.

(23) Matthews, B. W.; Colman, P. M.; Jansonius, J. N.; Titani, K.; Walsh, K. A.; Neurath, H. Structure of Thermolysin. Nature 1972, 238, $41-43$.

(24) Matthews, B. W.; Jansonius, J. N.; Colman, P. M.; Schoenborn, B. P.; Dupourque, D. Three-Dimensional Structure of Thermolysin. Nature 1972, 238, 37-41.

(25) Vriend, G.; Eijsink, V. Prediction and analysis of structure, stability and unfolding of thermolysin-like proteases. J. Comput.-Aided Mol. Des. 1993, 7, 367-396.

(26) de Kreij, A.; Venema, G.; van den Burg, B. Substrate Specificity in the Highly Heterogeneous M4 Peptidase Family Is Determined by a Small Subset of Amino Acids. J. Biol. Chem. 2000, 275, 31115-31120.

(27) de Kreij, A.; van den Burg, B.; Veltman, O. R.; Vriend, G.; Venema, G.; Eijsink, V. G. H. The effect of changing the hydrophobic S1' subsite of thermolysin-like proteases on substrate specificity. Eur. J. Biochem. 2001, 268, 4985-4991.

(28) Berlier, J. E.; Rothe, A.; Buller, G.; Bradford, J.; Gray, D. R.; Filanoski, B. J.; Telford, W. G.; Yue, S.; Liu, J.; Cheung, C.-Y.; Chang, W.; Hirsch, J. D.; Beechem, J. M.; Haugland, R. P.; Haugland, R. P. Quantitative Comparison of Long-wavelength Alexa Fluor Dyes to Cy Dyes: Fluorescence of the Dyes and Their Bioconjugates. J. Histochem. Cytochem. 2003, 51, 1699-1712.

(29) Lakowicz, J. R. Principles of Fluorescence Spectroscopy, 3rd ed.; Springer: New York, 2006.

(30) Liu, Y.; Kati, W.; Chen, C.-M.; Tripathi, R.; Molla, A.; Kohlbrenner, W. Use of a Fluorescence Plate Reader for Measuring Kinetic Parameters with Inner Filter Effect Correction. Anal. Biochem. 1999, 267, 331-335.

(31) Palmier, M. O.; Van Doren, S. R. Rapid determination of enzyme kinetics from fluorescence: Overcoming the inner filter effect. Anal. Biochem. 2007, 371, 43-51.

(32) Case, A.; Stein, R. L. Mechanistic Origins of the Substrate Selectivity of Serine Proteases. Biochemistry 2003, 42, 3335-3348. 
(33) Morihara, K.; Tsuzuki, H. Thermolysin: Kinetic Study with Oligopeptides. Eur. J. Biochem. 1970, 15, 374-380.

(34) Nishino, N.; Powers, J. C. Pseudomonas aeruginosa elastase. Development of a new substrate, inhibitors, and an affinity ligand. $J$. Biol. Chem. 1980, 255, 3482-3486.

(35) Izquierdo, M. C.; Stein, R. L. Mechanistic studies of thermolysin. J. Am. Chem. Soc. 1990, 112, 6054-6062.

(36) Sato, E.; Hattori, H.; Kanaoka, Y. Fluorogenic Bimane Substrates with Dabsyl Group for Endopeptidases: Chymotrypsin, Collagenase and Thermolysin. J. Pharmacobio-Dyn. 1991, 14, 599604.

(37) Hartmann, A.; Krainer, G.; Schlierf, M. Different Fluorophore Labeling Strategies and Designs Affect Millisecond Kinetics of DNA Hairpins. Molecules 2014, 19, 13735.

(38) Hoefling, M.; Lima, N.; Haenni, D.; Seidel, C. A. M.; Schuler, B.; Grubmüller, H. Structural Heterogeneity and Quantitative FRET Efficiency Distributions of Polyprolines through a Hybrid Atomistic Simulation and Monte Carlo Approach. PLoS One 2011, 6, No. e19791.

(39) Hughes, L. D.; Rawle, R. J.; Boxer, S. G. Choose Your Label Wisely: Water-Soluble Fluorophores Often Interact with Lipid Bilayers. PLoS One 2014, 9, No. e87649.

(40) Dimura, M.; Peulen, T. O.; Hanke, C. A.; Prakash, A.; Gohlke, H.; Seidel, C. A. M. Quantitative FRET studies and integrative modeling unravel the structure and dynamics of biomolecular systems. Curr. Opin. Struct. Biol. 2016, 40, 163-185.

(41) Miller, M. A.; Barkal, L.; Jeng, K.; Herrlich, A.; Moss, M.; Griffith, L. G.; Lauffenburger, D. A. Proteolytic Activity Matrix Analysis (PrAMA) for simultaneous determination of multiple protease activities. Integr. Biol. 2011, 3, 422-438.

(42) Chen, C.-H.; Miller, M. A.; Sarkar, A.; Beste, M. T.; Isaacson, K. B.; Lauffenburger, D. A.; Griffith, L. G.; Han, J. Multiplexed Protease Activity Assay for Low-Volume Clinical Samples Using Droplet-Based Microfluidics and Its Application to Endometriosis. J. Am. Chem. Soc. 2013, 135, 1645-1648.

(43) Rane, T. D.; Zec, H. C.; Wang, T.-H. A Barcode-Free Combinatorial Screening Platform for Matrix Metalloproteinase Screening. Anal. Chem. 2015, 87, 1950-1956.

(44) Ng, E. X.; Miller, M. A.; Jing, T.; Chen, C.-H. Single cell multiplexed assay for proteolytic activity using droplet microfluidics. Biosens. Bioelectron. 2016, 81, 408-414.

(45) Blank, K.; De Cremer, G.; Hofkens, J. Fluorescence-based analysis of enzymes at the single-molecule level. Biotechnol. J. 2009, 4, 465-479.

(46) Turunen, P.; Rowan, A. E.; Blank, K. Single-enzyme kinetics with fluorogenic substrates: lessons learnt and future directions. FEBS Lett. 2014, 588, 3553-3563.

(47) Fischer, R.; Bächle, D.; Fotin-Mleczek, M.; Jung, G.; Kalbacher, H.; Brock, R. A Targeted Protease Substrate for a Quantitative Determination of Protease Activities in the Endolysosomal Pathway. ChemBioChem 2006, 7, 1428-1434.

(48) Copolovici, D. M.; Langel, K.; Eriste, E.; Langel, Ü. CellPenetrating Peptides: Design, Synthesis, and Applications. ACS Nano 2014, 8, 1972-1994.

(49) Van Den Burg, B.; Eijsink, V. G. H.; Stulp, B. K.; Venema, G. One-step affinity purification of Bacillus neutral proteases using bacitracin-silica. J. Biochem. Biophys. Methods 1989, 18, 209-219. 